Anaesthesist 2011 · 60:705-706 DOI 10.1007/s00101-011-1929-5

Online publiziert: 11. August 2011

(c) Springer-Verlag 2011

\author{
P. Meybohm \\ Klinik für Anästhesiologie und Operative Intensivmedizin, \\ Universitätsklinikum Schleswig-Holstein, Campus Kiel
}

\title{
Perioperative Risikostratifizierung mithilfe von Biomarkern
}

\author{
"Science or fiction?"
}

Trotz des intensiven medizinischen Fortschritts in den vergangenen Jahren treten heute immer noch bei einem von 8 nichtherzchirurgischen Patienten (ca. 10-15\%) perioperativ potenziell lebensbedrohliche kardiovaskuläre Komplikationen wie akuter Myokardinfarkt, Schlaganfall, akute Herzinsuffizienz oder maligne Herzrhythmusstörung auf [1]. Die Ursachen sind multifaktoriell. Dabei spielt die Art des chirurgischen Eingriffs, aber auch das individuelle Risiko des Patienten, bestehend aus Patientenalter, Begleiterkrankungen und linksventrikulärer Pumpfunktion, eine entscheidende Rolle. Um potenzielle Risikopatienten bereits präoperativ zu identifizieren, wurde in den vergangenen Jahren eine Reihe klinischer Risiko-Scores zur perioperativen kardiovaskulären Risikostratifizierung entwickelt $[2,3,4]$, eine präoperative Vorhersage der postoperativen Morbidität oder Letalität für den Einzelnen im Alltag ist damit jedoch nicht oder nur sehr eingeschränkt möglich.

Zusätzliche präoperative technische Voruntersuchungen oder Laboranalysen könnten hierbei neben der präoperativen Anamnese eine interessante Option darstellen, um die Prädiktion des individuellen Risikos für eine kardiovaskuläre Komplikation weiter zu verbessern. Bei der heute vorliegenden Vielfalt an technischen und laborchemischen Untersuchungen bringt seit dem vergangenen Jahr eine gemeinsame Empfehlung der Deut- schen Gesellschaft für Anästhesiologie und Intensivmedizin (DGAI), der Deutschen Gesellschaft für Chirurgie (DGCH) und der Deutschen Gesellschaft für Innere Medizin (DGIM) nun „Licht in das Dunkel“ und gibt disziplinübergreifend detaillierte Handlungsempfehlungen zur präoperativen Evaluation erwachsener Patienten vor elektiven, nichtkardiochirurgischen Eingriffen [5]. Insbesondere bei Patienten mit bekannten oder vermuteten kardiovaskulären Vorerkrankungen kann präoperativ eine differenzierte kardiologische Abklärung erforderlich sein. Die Indikation hierfür ist allerdings wegen des vielfach unklaren Nutzens präoperativer kardiologischer oder herzchirurgischer Interventionen im Hinblick auf die perioperative Morbidität und Letalität streng zu stellen.

Vor diesem Hintergrund stellt sich die Frage, ob vor einem elektiven Eingriff kardiale Biomarker als objektive Hilfsmittel bei der Beurteilung des individuellen kardiovaskulären Risikos hilfreich sind.

Schlitt et al. diskutieren in ihrem Beitrag den aktuellen Stellenwert kardialer Biomarker in der perioperativen Medizin. Demnach kann die präoperative Bestimmung von kardialen Integritätsmarkern helfen, kardiovaskuläre Risikopatienten zu identifizieren. So weisen etwa Patienten mit einer präoperativ erhöhten Konzentration des N-terminalen Fragments des natriuretischen Peptids vom BTyp (NT-proBNP) perioperativ ein bis zu 20-fach erhöhtes Risiko für kardiovaskuläre Komplikationen auf [6]. Die Autoren empfehlen daher, bei Patienten mit Herzinsuffizienz und einer geplanten Operation mit einem mittleren/hohen Risiko für kardiovaskuläre Komplikationen (z. B. gefäßchirurgische Eingriffe, intraabdominelle oder intrathorakale Eingriffe, orthopädische Eingriffe) präoperativ NTproBNP zu bestimmen. Bei pathologisch erhöhten Werten sollte dann eine präoperative internistische (kardiologische) Vorstellung erwogen werden. Aufgrund fehlender prospektiver Studien konnten die Autoren zwar (noch) keinen praktischen Handlungsablauf im Sinne eines leitliniengerechten Vorgehens definieren, stellen jedoch ihr hausinternes Vorgehen im Sinne eines alltagstauglichen Algorithmus vor.

Die zweite, klinisch aber wesentlich spannendere Frage lautet, inwieweit kardiale Biomarker bei kardiovaskulären Risikopatienten einen Beitrag zur Senkung der perioperativen Morbidität oder Letalität leisten können. Auch hier gilt, dass prospektive klinische Untersuchungen, die eine Senkung der perioperativen Komplikationen durch die laborchemische Bestimmung von Biomarkern präoperativ aufzeigen, bislang fehlen.

Die Empfehlungen der Autoren bezüglich des konkreten Einsatzes der Biomarker bleiben insofern - nachvollziehbar vage. Als Konsequenz sind daher zukünftig Studien unverzichtbar, in denen nach 
erfolgreicher Identifikation von Hochrisikopatienten dann in einem zweiten Schritt mithilfe eines geeigneten Biomarkers bereits präoperativ eine gezielte Therapie der Begleiterkrankungen und eine optimierte perioperative Betreuung mit dem Ziel der Senkung von kardiovaskulären Komplikationen prospektiv untersucht werden.

In den vergangenen Jahren haben verschiedenste perioperative Interventionsstudien mit dem Ziel der Verbesserung des postoperativen Outcomes (z. B. zielorientierte hämodynamische Optimierung [7], Periduralanästhesie, intensivierte Insulintherapie [8] oder prophylaktische $\beta$-Rezeptoren-Blockertherapie [9] etc.) eher kontroverse Ergebnisse hervorgebracht. Die Gründe hierfür sind multifaktoriell. Ein Erklärungsansatz könnte aber sein, dass auch Studien zu im Grundsatz vielversprechenden Therapieansätzen wegen fehlender Risikostratifizierung und inadäquater Indikationsstellung häufig (zu) viele „gesunde“ Patienten aufnehmen und damit an statistischer Aussagekraft verlieren. Insofern kommt der präoperativen Identifikation der eigentlichen Hochrisikopatienten, z. B. anhand von kardialen Biomarkern, die entscheidende Bedeutung zu.

Darüber hinaus sollten in zukünftigen Studien auch noch folgende Fragen beantwortet werden:

a) Welchen Stellenwert und welchen zusätzlichen Nutzen hat die präoperative Bestimmung von kardialen Biomarkern, insbesondere im Bezug zu den bisher vorhandenen verschiedenen Diagnostik-Tools (z. B. 12-Kanal EKG, Echokardiographie)?

b) Welcher Marker soll zu welchem Zeitpunkt unter welchen Umständen bei welchen Patienten bestimmt werden?

In diesem Zusammenhang ist das aktuelle Bestreben der DGAI zu begrüßen, kooperative klinische Studien in unserem Fachbereich zu fördern. Die DGAI hat hierzu vor Kurzem eine Kommission für klinische Studien (KKF) installiert und einen ersten Aufruf an alle Lehrstuhlinhaber sowie alle Mitglieder der Programmkommission verschickt, sodass interessante Themenfelder identifiziert und relevante Fragestellungen in der perioperativen
Medizin gemeinsam angegangen werden können.

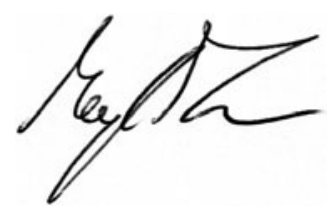

P. Meybohm

\section{Korrespondenzadresse}

\section{PD Dr. P. Meybohm}

Klinik für Anästhesiologie und Operative Intensivmedizin, Universitätsklinikum Schleswig-Holstein, Campus Kiel Arnold-Heller-Str. 3, 24105 Kiel meybohm@anaesthesie.uni-kiel.de

\section{Literatur}

1. Choi JH, Cho DK, Song YB et al (2010) Preoperative NT-proBNP and CRP predict perioperative major cardiovascular events in non-cardiac surgery. Heart 96:56-62

2. Goldman L, Caldera DL, Nussbaum SR et al (1977) Multifactorial index of cardiac risk in noncardiac surgical procedures. N Engl J Med 297:845-850

3. Lee TH, Marcantonio ER, Mangione CM et al (1999) Derivation and prospective validation of a simple index for prediction of cardiac risk of major noncardiac surgery. Circulation 100:1043-1049

4. Kertai MD, Boersma E, Klein J et al (2005) Optimizing the prediction of perioperative mortality in vascular surgery by using a customized probability model. Arch Intern Med 165:898-904

5. Deutsche Gesellschaft für Anästhesiologie und Intensivmedizin, Deutsche Gesellschaft für Chirurgie und Deutsche Gesellschaft für Innere Medizin (2010) Präoperative Evaluation erwachsener Patienten vor elektiven, nichtkardiochirurgischen Eingriffen. Anaesthesist 59:1041-1050

6. Ryding $A D$, Kumar $S$, Worthington $A M$, Burgess $D$ (2009) Prognostic value of brain natriuretic peptide in noncardiac surgery: a meta-analysis. Anesthesiology 111:311-319

7. Gurgel ST, do Nascimento P Jr (2011) Maintaining tissue perfusion in high-risk surgical patients: a systematic review of randomized clinical trials. Anesth Analg 112:1384-1391

8. Kao LS, Meeks D, Moyer VA, Lally KP (2009) Perioperative glycaemic control regimens for preventing surgical site infections in adults. Cochrane Database Syst Rev:CD006806

9. Preckel B, Poels M, Wappler F et al (2010) Perioperative $\beta$-Rezeptorenblockade - Für und wider. Anaesthesist 59:643-651

\section{Neue Leitlinie zur Versorgung Schwerverletzter}

Die Deutsche Gesellschaft für Unfallchirurgie veröffentlicht mit 10 weiteren medizinischen Fachgesellschaften die neue S3Leitlinie „Polytrauma/Schwerverletzten-Behandlung". Erstmalig werden Qualitätsstandards für die Akutversorgung Schwerverletzter beschrieben. Die Leitlinie ist eine Entscheidungshilfe für alle an der Unfallversorgung beteiligten Berufsgruppen.

Jährlich verunglücken in Deutschland bei Unfällen 8 Millionen Menschen. Für Unfallchirurgen ist insbesondere die Versorgung von Patienten mit Mehrfachverletzungen eine verantwortungsvolle Herausforderung. Sie macht eine fachübergreifende Versorgung und Zusammenarbeit notwendig. Dazu haben alle für die Notfallversorgung relevanten medizinischen Fachdisziplinen mit nahezu 100 Experten den aktuellen Stand der wissenschaftlichen Erkenntnisse strukturiert zusammengetragen. Die Leitlinie auf S3-Niveau bietet eine rationale und transparente Basis für die optimale Therapie in der Akutphase. Die Empfehlungen sollen zur Verbesserung der Struktur- und Prozessqualität in den Kliniken sowie in der präklinischen Versorgung beitragen und durch deren Umsetzung die Ergebnisqualität, gemessen an der Sterblichkeit oder Lebensqualität, verbessern helfen.

Die Leitlinie ist Teil der "Initiative Qualität und Sicherheit in Orthopädie und Unfallchirurgie" und bildet mit dem TraumaNetzwerk DGU das Zentrum der qualitätsgesicherten Versorgung von Schwerverletzten in Deutschland.

Die Leitlinie ist veröffentlicht auf: www.awmf.org.

Quelle: Deutsche Gesellschaft für Unfallchirurgie, Berlin, www.dgu-online.de 